\title{
AND THE WINNER IS... ALGUNAS REFLEXIONES QUE PUEDEN LLEVAR A UNA VISIÓN MÁS AJUSTADA DE LA CIENCIA ${ }^{1}$
}

\author{
Eulalia PÉREZ SEDEÑO \\ Dpt. Lógica y Filosofia de la Ciencia UPV/EHU \\ ylxpesee@sf.ehu.es
}

La publicación de The Structure of Scientific Revolutions en 1962, por T.S. Kuhn, encendió la mecha de una rebelión contra la filosofía de la ciencia, que acabó siendo de la propia filosofía de la ciencia, aunque antes ya se habían alzado voces que indicaban lo que se avecinaba. Las críticas que los rebeldes efectuasen a la filosofía de la ciencia «ortodoxa", también conocida como la concepción heredada, iban dirigidas fundamentalmente a la manera de encarar los problemas. Si la filosofía de la ciencia utiliza la lógica como instrumento de análisis, argumentaban, sus resultados tendrían poco valor informativo: la lógica se ocupa de la forma, no del contenido, por lo que mediante su aplicación sólo se llegaría a enunciados válidos para todas las ciencias posibles; por ejemplo, se podría averiguar la forma lógica de todas las explicaciones científicas posibles, de todas las leyes lógicas posibles, se conseguiría averiguar cuál es la estructura lógica de todas las teorías. La filosofía de la ciencia versaría sobre la relación lógica existente entre las hipótesis y los datos empíricos que la apoyan, pero no se ocuparía de lo que realmente tiene valor informativo: las explicaciones científicas concretas, las leyes y teorías particulares etc. Además, los "heterodoxos" le reprochaban a la filosofía de la ciencia que se limitara a la parte estática, dejando a un lado un aspecto fundamental: su práctica y desarrollo. Si se quería realizar un análisis adecuado y completo de la ciencia había que sustituir el método lógico por el histórico, unir al análisis de los libros de texto el de las actividades que se llevan a cabo en el laboratorio. C08-02.

1 Este trabajo ha sido realizado en parte dentro del proyecto de investigación PB98-0495- 
Pero no era éste el único reproche que le hacían a la filosofía de la ciencia. Sus supuestos empiristas tampoco escapaban a las críticas. Consideraban, por ejemplo, que la idea de que todas las ciencias que se ocupan de la realidad tienen una base empírica-observacional ha conducido a muchos errores. Hanson fue uno de los primeros en formular serias críticas a la dicotomía lenguaje teórico/lenguaje observacional al hablar del "tinte teórico" —o la "carga teórica" como se dice habitualmente- de todas las observaciones. Según esa idea no hay un observador neutral, alguien que no observe "con los ojos de una teoría".

Las críticas a algunos de los supuestos básicos del empirismo lógico y la irrupción de los nuevos enfoques historicistas produjo una renovación en la disciplina con diversas manifestaciones características ${ }^{2}$. Nuevos modelos para la estructura y cambio científicos, el análisis de las formas argumentativas en la justificación de hipótesis o de problemas como la inconmensurabilidad o la infradeterminación, se originaron o cobraron nuevo ímpetu a partir del giro historicista. Pero, al rechazar al excesivo optimismo positivista, algunos autores extrajeron consecuencias sumamente extremas que condujeron a enfoques relativistas que ponían en duda e incluso negaban tajantemente la posibilidad de hallar o idear criterios objetivos de evaluación y elección ${ }^{3}$. Tras el desafío relativista y el rechazo al positivismo uno de los retos más importante fue, y sigue siendo, lograr una nueva caracterización del progreso y de la racionalidad y objetividad científica.

Todas estas cuestiones y la preocupación por la imposibilidad de enunciar criterios universales de racionalidad no desembocaron en un nihilismo epistemológico sino, por el contrario, están en la base del proyecto naturalista que se ha generalizado durante los años $90^{4}$. Se puede caracterizar el naturalismo como la postura filosófica que sostiene que todo lo que existe u ocurre en el mundo es natural, en el sentido de que requiere una explicación mediante los

2 Hay diversas obras que examinan y evalúan el camino recorrido desde la publicación de la obra de Kuhn. Véase, por ejemplo, DuRAN (1998), KLEE (1997) y Solfs (1998).

3 El Programa Fuerte propuesto en la Unidad de Estudios sobre Ciencia de la Universidad de Edimburgo a finales de los años 70 provocó un agrio enfrentamiento entre filósofos racionalistas y sociólogos relativistas. Pese a lo crudo de la provocación sociológica y lo airado de la respuesta filosófica, el análisis teórico y el estudio de casos derivado de los programas de Edimburgo y Bath son de importancia indiscutible para la filosofía actual de la ciencia.

4 El programa se origina con el artículo de QUINE (1970), aunque el "primer manifiesto" es Giere (1985). Véase, Ambrogi (1999). 
métodos de las ciencias naturales. La naturalización del estudio de la ciencia ha de entenderse, entonces, como un intento de resolver mediante la ciencia las tradicionales cuestiones filosóficas sobre el descubrimiento, desarrollo y justificación de las ideas científicas. El análisis filosófico se convierte así en continuo respecto al análisis científico (aunque en las versiones más radicales, desaparece absorbido por éste). Se considera que tanto la producción como la evaluación de teorías científicas son procesos naturales desarrollados por individuos con determinadas capacidades cognitivas puestas en funcionamiento en ambientes sociales, es decir, en colaboración con otros científicos, instituciones y público en general. La psicología, la biología y la sociología se convierten, entonces, en instrumentos fundamentales del estudio naturalizado de la ciencia y la tarea de reelaborar las nociones de racionalidad y objetividad en el nuevo marco naturalista, evitando las versiones fuertes de relativismo, ocupa gran parte de las discusiones en la actualidad ${ }^{5}$.

La filosofía de la ciencia naturalizada ofrece una imagen mucho más contextualizada de la empresa científica de lo que había sido habitual en las concepciones tradicionales. Al analizar la actividad científica en un marco semejante, obtenemos un conocimiento científico situado, cuya aparición y aceptación depende en parte de condiciones particulares del propio científico y del contexto profesional y social en el que desarrolla su labor. Entre estos factores tradicionalmente considerados "extracientíficos» que condicionan la génesis y validación de teorías científicas, el género es uno de los que ha despertado mayor interés en los últimos años. Los análisis de las relaciones entre la ciencia y el género proporcionan argumentos y casos de estudio para el debate general acerca del papel de valores no epistémicos en ciencia y para una nueva noción de objetividad.

Aparentemente, parece haber una cierta incongruencia en hablar de filosofía de la ciencia y feminismo: en efecto, la filosofía de la ciencia se ocupa del conocimiento científico ${ }^{6}$, por ejemplo de sus condiciones de posibilidad, de

5 La literatura generada per este enfoque es inmensa, por lo que no haré ninguna cita. Remito a uno de los pocos trabajos publicados en nuestro país, AMBROGI (ed.) (1999) que contiene una buena y larga introducción a esta corriente y abundante bibliografia.

- En las últimas décadas, y dado entre otras cosas al desarrollo de los estudios de ciencia, tecnología y sociedad, se ha extendido el interés a la tecnología. Aunque aquí me limitaré a hablar de ciencia, debe entenderse que básicamente todo lo que aquí expongo se puede aplicar con ligeras variaciones a la tecnología, más aún cuando se aboga, como se verá más adelante, por una concepción del conocimiento no meramente proposicional y por la no aceptación de la separación entre ciencia y política, que en el caso de la tecnología es más claro aún. Véase PÉREZ SEDEÑo (1998b). 
los criterios que permiten decidir cúando nuestras creencias son "científicas" o "verdaderas" (sea lo que fuere que entendemos por esto), sobre la naturaleza de la verdad, etc. y el feminismo (en sus diferentes versiones) es una práctica/actividad sociopolítica que pretende eliminar la desigualdad por razones de sexo.

El interés por la interacción entre género y ciencia, y por la relevancia de la variable género para el análisis filosófico de la actividad científica, surge de investigaciones feministas acerca del escaso número de mujeres en la historia de la ciencia y las barreras institucionales y sociopsicológicas que han obstaculizado y siguen obstaculizando el acceso de las mujeres a la ciencia y la tecnología. Estas investigaciones tuvieron como resultado la recuperación de figuras femeninas olvidadas por la historia de la ciencia tradicional y la identificación de patrones de discriminación explícitos e implícitos, así como la propuesta de transformaciones pedagógicas destinadas a fomentar el estudio de disciplinas científicas y tecnológicas por parte de las mujeres ${ }^{7}$.

Pero las cuestiones históricas y sociológicas relativas a los obstáculos que han impedido una mayor presencia de mujeres en las prácticas científico-tecnológicas poseen también una relevancia especial para la filosofía de la ciencia: ¿en qué medida esta ausencia socialmente condicionada ha tenido algún tipo de impacto sobre problemas, métodos, contenidos teóricos y desarrollos tecnológicos? ¿sería diferente nuestra ciencia en el caso de que hubiera habido más mujeres practicándola? Estas preguntas tienen implicaciones que trascienden los intereses feministas para abordar de un modo directo el problema de la racionalidad y la objetividad científica que se deriva de asumir el carácter situado del conocimiento y la necesidad de contextualizar los múltiples aspectos de las prácticas científicas.

Los trabajos de un buen número de autoras feministas han mostrado los sesgos de género que aparecen en muchas disciplinas científicas, bien en afirmaciones concretas, bien en sus principios metodológicos o su lenguaje. Diversos estudios sociológicos, históricos y antropológicos sobre distintas ciencias y teorías particulares se han ocupado de señalar cómo valores contextuales ligados a preconcepciones de género guían la investigación, determinan qué hipótesis seleccionar, qué método de contrastación vamos a usar...; limitan, en

7 Véase, por ejemplo, Pérez Sedeño (1998a), (2000), Gonzalez Garcla y Pérez Sedeño (2000) para un repaso más amplio de todos estos aspectos y la bibliografía más relevante. 
suma, qué vamos a aceptar como conocimiento científico justificado. Pero también los aspectos teórico-conceptuales reproducen la ideología de género, como revelan los trabajos realizados sobre el papel de las metáforas de género en disciplinas como la primatología (Haraway, 1989), la etología (Bleier, 1984) o la biología humana (Fausto-Sterling, 1985). Evelyn Fox Keller (1995) ha mostrado, por ejemplo, cómo la molécula rectora dirigió la investigación en biología celular, biología evolutiva y genética; y Emily Martin (1991) ha analizado el lenguaje médico y biológico que describe el óvulo y el esperma de tal modo que las metáforas usadas constituyen estereotipos de nuestras definiciones de lo masculino y femenino y contribuyen a reforzarlos.

En lo que respecta a las ciencias sociales, diversas sociólogas feministas examinaron los sesgos sexistas y androcéntricos en sus disciplinas. Dorothy $\mathrm{Smith}$ (1988), por ejemplo, mantiene que hombres y mujeres tienen experiencias diferentes del mundo, pues los hombres encuentran un mundo ya procesado, lo que no sucede en el caso de las mujeres. Incluso parte de la literatura feminista defiende que fundamentar las teorías de las ciencias sociales en el «lenguaje y la experiencia reales" de las propias mujeres sería más adecuado. Se considera (en línea con los enfoques del punto de vista que se tratarán más adelante) que dichas experiencias proporcionan un acceso a verdades del mundo social a las que no pueden acceder los varones y que serían la piedra de toque contra la que se contrastarían las hipótesis, ya que dicha experiencia «es un tipo de experiencia social más completa y menos distorsionada". Afirmaciones semejantes se basan en las diferentes posiciones sociales ocupadas por mujeres y hombres $y$, sobre todo, el distinto poder detentado por ellos, factores que estructuran las relaciones humanas de todo tipo. Se afirma que las diferencias de género organizan las creencias y experiencias personales y que los varones son, por lo general, socialmente dominantes. Como consecuencia, ha habido una tendencia a generalizar a partir de las experiencias de los varones, despreciando diferencias de género potencialmente significativas; por ello se considera que las ciencias sociales constituyen una expresión de las experiencias masculinas que se presentan como las experiencias de toda la especie humana y es en ese sentido en el que se habla de una ciencia "masculina".

El examen crítico de la historia de la filosofía también ha resultado crucial a la hora de mantener el carácter ideológicamente masculino de determinados conceptos epistemológicos relevantes para el análisis filosófico de la ciencia. Filósofas como Genevieve Lloyd (1984) han mostrado cómo conceptos como el de racionalidad se derivan de estereotipos masculinos, y ha trazado la asociación 
entre racionalidad y masculinidad de Platón a Kant y Hegel. La masculinización de la razón a través de metáforas ha sido objeto de análisis por parte de Phyllis Rooney $(1995)^{8}$. Asimismo, la psicología educativa ha sugerido que la forma de enfrentar y resolver problemas puede ser diferente según el género y que las normas de conducta de cada género podrían incluir la adquisición de normas cognitivas de género.

En definitiva, se han encontrado en las ciencias diversos tipos de manifestaciones del efecto de la exclusión de las mujeres de sus prácticas. En primer lugar, se han atribuido a menudo capacidades cognitivas, al menos en su forma más desarrollada, a los varones, de tal modo que su expresión en las mujeres se considera impropia. En segundo lugar, en las ciencias naturales persisten patrones descriptivos y explicativos que son androcéntricos y sesgados por el género. En tercer lugar, en las ciencias sociales y de la conducta los modos de teorizar son de tal tipo que las mujeres se vuelven invisibles en cuanto agentes cognitivos y actrices sociales, por lo que su subordinación se considera inevitable y natural.

La pregunta que se hacen las feministas es qué tipo de concepción de la ciencia apoya o facilita estos análisis. Lo cual hace que se replanteen la adecuación de la visión racionalista estrecha de la concepción heredada, según la cual el conocimiento cientifico se caracteriza por su objetividad, por su neutralidad, porque sus contenidos carecen de valores, así como la idea de la tajante distinción entre contexto de descubrimiento y contexto de justificación, que cierra el paso a la categoría de género ${ }^{9}$. Se ponen seriamente en cuestión las tesis según las cuales el método científico se distingue precisamente por la búsqueda desinteresada de la verdad mediante la formulación de hipótesis que son contrastadas por medio de técnicas muy elaboradas (experimentación y repetición de experimentos controlados, uso de técnicas cuantitativas sofisticadas, crítica por parte de la comunidad científica); que el hecho de que esas hipótesis sean sometidas a muy diferentes y numerosas pruebas hace que el producto final obtenido, el conocimiento científico, se considere libre de errores, que

8 Por supuesto, el análisis y crítica de la noción de objetividad científica constituye una parte importante en este terreno, pero de ello me ocuparé más adelante.

9 Insisto en que este tipo de críticas no proceden sólo del ámbito feminista, sino que es compartido por otros muchos autores, aunque éstos no insistan (ni incluyan a veces) la categoría "género" como una a tener en cuenta. 
se introducirían en él si no se dejaran fuera de su ámbito factores tales como los sentimientos, los compromisos políticos o las preferencias estéticas. Se duda seriamente, cuando no se niega rotundamente, de la afirmación de que la ciencia está libre de valores, que significa que los valores contextuales y los valores constitutivos o internos son distintos e independientes entre sí, a la vez que se mantiene que los valores contextuales no desempeñan ningún papel en el funcionamiento interno de la investigación, esto es, en la observación, experimentación, y en los razonamientos que permiten justificar una hipótesis o una teoría.

Y se pasa a abordar qué concepciones pueden obstaculizar los movimientos sexistas; si hay conceptos de justificación que muestren por qué las representaciones del género que se encuentran en las ciencias sociales, comportamentales y naturales parecen correctas y por qué no; si es posible repensar los conceptos de verdad, racionalidad, objetividad, etc., de manera que se puedan eliminar de ellos los sesgos de género, etc. A todas estas cuestiones y a otras muchas pretende responder la filosofía de la ciencia hecha desde una perspectiva feminista y las diversas epistemologías feministas en las que se apoya. Porque una vez se introduce el género en estos análisis, vemos cómo cambia la consideración de muchas cuestiones. En efecto, hay que insistir en que la perspectiva feminista complementa los instrumentos conceptuales necesarios para criticar la concepción de la ciencia que entra en crisis en las últimas décadas. Pero, aunque las autoras feministas tienen en común el reconocimiento de la relevancia de valores contextuales (en particular, los relacionados con el género) para la aparición y evaluación de teorías científicas, entienden esta relevancia de maneras distintas.

Las denominadas teóricas del "punto de vista" (standpoint theorists), cuyo enfoque se enmarca en la tradición marxista, consideran fundamental dar cuenta de las diversas condiciones materiales e históricas en que las personas producen conocimiento. El conocimiento autoritario, adecuado a las normas, procede de una parte muy concreta y pequeña de la población: varones blancos, educados y de clase media. En consecuencia, las mujeres se hallan en una posición epistémica marginal y oprimida. Pero esa posición se puede convertir en una de privilegio ya que las clases dominadas o excluidas pueden entender de manera más objetiva el orden social por no tener interés personal en su mantenimiento. Además, el conocimiento que la mujer tiene de la cultura dominante, de la masculina, y de sus propias experiencias y percepciones necesariamente desviadas la dará una mayor amplitud de conocimientos. La 
consecuencia obvia, para muchas teóricas, es que la investigación científica hecha por mujeres incorporará este punto de vista privilegiado, lo que repercutirá en la identificación de problemas, así como la atención a datos, relaciones, modelos teóricos o alternativas metodológicas invisibles desde la perspectiva masculina dominante. Sin embargo, hay varios problemas . En primer lugar, jpor qué suponer que las mujeres -en posición de marginalidad, exclusión y opresión - poseen auténtico conocimiento de la cultura masculina dominante? En segundo, no hay que olvidar que las propias experiencias de las mujeres se dan en esa cultura masculina y que no tenemos evidencia suficiente que avale un modo de conocer único y común a todas las mujeres, sino, más bien, lo contrario. Además, la idea de que las mujeres tienen capacidades cognitivas distintivamente válidas es similar a la esgrimida para otros grupos, ya sea la comunidad negra o el proletariado, lo que plantea la pregunta de cuál de estas posiciones puede considerase con un mayor privilegio epistémico. No hay que olvidar que tan importantes como el género pueden ser otras variables y que para tener una comprensión global y cabal de la ciencia, las perspectivas han de ser múltiples, por lo que, como determinadas feministas postmodernistas proponen, habría que reemplazar la política del género o del sexo por una pluralidad de diferencias en donde el género perdería su posición privilegiada (Haraway, 1995).

Otro enfoque para la comprensión de las relaciones entre género y ciencia es la perspectiva psicodinámica de E. Fox Keller. Según dicha autora, el sentido de identidad del científico o científica en cualquier disciplina ha dependido de la internalización de las dicotomías de sujeto/objeto, fundamentales en el pensamiento masculino. Huellas de esas dicotomías, que poseen además una estructura jerárquica, se encuentran en muchos supuestos fundamentales de las disciplinas académicas tradicionales y se basan en el supuesto previo de la relación, también dicotómica y jerárquica, entre masculino/ femenino: por ejemplo público/privado, político/personal, razón/sentimiento, justicia/cuidado, poder/amor, etc. Pero además, Fox Keller (1983) mantiene que es posible hacer una ciencia feminista, es decir, una ciencia diferente a la que hacen los varones, en el método y en la forma de aproximación al objeto de estudio. Tal es su tesis en la biografía de Barbara McClintock, premio Nobel de Medicina y Fisiología en 1983, donde señala que McClintock tenía una manera de investigar que no diferenciaba (ni jerarquizaba) claramente entre objeto/sujeto, como es tradicional en la ciencia, sino que sentía una cierta empatia-simpatía con/por el material investigado. 
En otro punto del espectro tenemos el feminismo postmodernista, con autoras como Donna Haraway, que efectúa una de las críticas más radicales y prominentes. Aplica los métodos de investigación de los «estudios culturales" a la ciencia en un intento por convertir el postmodernismo en ciencia normal. Por un lado, Donna Haraway (1989) analiza los sesgos masculinos que se dan en los métodos que se utilizan para interpretar la conducta de los primates (ya sea en estado salvaje, ya en cautividad) en los foros en los que se habla y discute profusamente sobre ellos, y que van de la filosofia a los anuncios publicitarios: la dicotomía naturaleza/cultura es un resultado de la dominación humana que se proyecta al mundo animal. Por otro (Haraway, 1991, 1997), utiliza la imagen del cyborg, un híbrido de ser humano, computador y animal, para señalar la necesidad de una nueva ontología en un mundo en que se desarrollan a velocidad vertiginosa las nuevas tecnologías genéticas y neurocomputacionales que han difuminado los límites ontológicos tradicionales entre lo orgánico y lo mecánico, entre lo innato y lo medioambiental ${ }^{10}$.

Pero son los enfoques denominados empirismos feministas los que mayor relevancia e incidencia han tenido en el seno de la filosofía de la ciencia ya que entroncan con la tradición de la concepción heredada y han analizado los sesgos de género en instancias concretas de prácticas y teorías científicas. Específicamente, es posible encontrar sesgos sexistas y androcéntricos en aquellas disciplinas cientificas que se ocupan de investigar las diferencias entre hombres y mujeres (biología, psicología...), así como aquéllas que incorporan en sus elaboraciones teóricas ciertas preconcepciones acerca de tales diferencias (paleoantropología, arqueología, primatología...). Las teorías que dan cuenta de la evolución humana o el estudio biológico y psicológico de las diferencias sexuales en conductas o habilidades cognitivas (y sus causas) son importantes casos de estudio que muestran la relevancia del género para entender la aparición y la aceptación de determinadas teorías científicas, así como el hecho de que la presencia de investigadoras con un compromiso feminista permita la identificación de estos sesgos (de acuerdo, en este caso, con lo mantenido por las teóricas del punto de vista).

Los diferentes tipos de empirismos feministas se distinguen en la interpretación que ofrecen para estos sesgos sexistas y androcéntricos. Las "empiristas

10 No pretendo hacer aquí un repaso exhaustivo de las distintas tendencias feministas en filosofía de la ciencia, sobre lo que se puede encontrar abundante bibliografía. Véase, por ejemplo, Gómez (1999), González y Pérez Sedeño (2000), Pérez Sedeño (1995). 
ingenuas" sostienen que tales sesgos son simplemente eliminables con una mejor aplicación del método científico, de tal modo que se deje la ciencia libre de perniciosas influencias externas. Esta interpretación es compatible con la concepción tradicional de la ciencia como empresa neutral y autónoma, racional y objetiva, de descubrimiento de verdades, lo que ha suscitado críticas por parte de otras autoras feministas para quienes tal concepción no es más que una quimera positivista. Los argumentos que apuntan hacia el carácter situado y la contextualización del conocimiento científico parecen indicar que no es posible una ciencia neutral y libre de cualquier condicionamiento externo, han llevado a la articulación de "feminismos empiristas sofisticados", según los cuales las teorías científicas sexistas o androcéntricas no constituyen "mala ciencia" sino que son únicamente ejemplos del único tipo de ciencia que podemos tener, esto es, ciencia contextualizada. Por ese motivo, una de las tareas prioritarias es la revisión de la noción de ciencia subyacente.

Esa revisión se ha centrado, al menos, en tres puntos, aunque en absoluto privativos de las feministas. Por un lado, se ha investigado la complejidad de las relaciones existentes entre los supuestos básicos (background assumptions) de los científicos y su forma de practicar la ciencia ${ }^{11}$; se ha cuestionado la noción de objetividad ${ }^{12}$; y, por último y siguiendo la estela de Dewey, tambien se ha cuestionado la consideración del individuo -el sujeto cartesiano- como agente del conocimiento ${ }^{13}$. Esa indagación y crítica de tal concepción de la ciencia ha provocado el rechazo de la separación tajante entre ciencia y política, el reconocimiento de la importancia epistémica de los componenetes subjetivos del conocimiento científico y, finalmente, la reconfiguración del sujeto de conocimiento. También en este caso los argumentos y modelos ofrecidos varían entre las feministas ${ }^{14}$, pero me limitaré a examinar

11 Críticas que ya aparecen en Kuhn y Lakatos y que han continuado en la escritura feminista de mano de Lynn Nelson, Nancy Tuana o Helen Longino.

12 En especial la paración tajante entre objetividad y subjetividad, al igual que hicieran Feyerabend y Hanson y que han continuado Evelyn Fox Keller, Elizabeth Fee, Nancy Tuana o Lorraine Code.

13 Este es un punto crucial y de disputa entre los naturalismos cognitivistas y los sociologistas. Sin embargo, creo que se puede argumentar la complementariedad de ambos enfoques, sobre todo en aquellos modelos en que el sujeto cognoscente es el individuo en la comunidad.

14 Tampoco ellas - nosotras - nos vemos libres de los valores imbricados en nuestros supuestos básicos y, por tanto, la respuesta ofrecida depende de la teoría feminista de la que proceden: marxismo liberal, socialismo, postmodernismo, etc. Véase, por ejemplo, Sue V. RoSSER (1992) o Helen LONGINO (2000). 
las críticas y los argumentos que han llevado a esas posturas y luego expondré uno de los modelos a mi juicio más interesantes.

El rechazo a la dicotomía entre ciencia y política se basa en el examen que diversas autoras y autores han efectuado de distintas teorías que mostraban los sesgos de género en ellas ${ }^{15}$. En esta ocasión seguiré aquí parte de lo que se ha llamado «el problema de los sexos» tal y como fue abordado por la craneología en la segunda mitad del s. XIX ${ }^{16}$. Los craneólogos del s. XIX consideraban que sus teorías eran completamente objetivas, logradas a través de procedimientos inductivos y con datos cuantificables. Según éstas, las medidas de los cráneos, y su capacidad, mostraban la jerarquía de las razas y los sexos, en una escala lineal de valor intelectual que confirmaban los prejuicios del momento: «los negros, las mujeres y las clases pobres ocupan puestos subordinados debido a los rigurosos dictados de la naturaleza" (Gould, 1997, pág. 91).

Los índices clasificatorios que utilizaban los craneólogos, esto es, qué conjunto de rasgos constituían evidencia de superioridad y qué de inferioridad, variaban. A mitad del s. XIX, dados los problemas que planteaban algunos para mantener la inferioridad de las mujeres ${ }^{17}$, se comienzan a buscar otros. En un artículo publicado en 1869 , H. Schaaffhausen establece como características para establecer el índice clasificatorio las siguientes: la proyección de las protuberancias parietales, la menor elevación del hueso frontal, una base craneal más corta y estrecha, un arco dental más elíptico y la inclinación al prognatismo (ángulo facial pequeño). Estas y sólo éstas permitían indicar la etapa de evolución de una clase social y el desarrollo inferior de la mujer, puesto que, según Schaaffhausen, eran las características que poseían las mujeres y las razas no europeas. Es decir, el procedimiento «empírico» e «inductivo" utilizado era

15 Como dije antes, el objetivo prioritario lo han constituido las ciencias biosociales, debido al papel que desempeñan en el mantenimiento de la sociedad patriarcal. La bibliografía al respecto es enorme: por ejemplo, BLEIER, 1979 y 1984, CAPLAN (1978), FAUSTO-STERLING (1985), S. JaY GOULD (1981), JordANOVA (1990), HubBARD (1990), LEWONTIN et al. (1984), Laqueur (1990), Longino (1990) cap. 6 y 7, Longino y Doell (1983), Pérez Sedeño (1997), (1998), (1998a), RUSE (1980), SAYERS (1982), SCHIEBINGER (1989), (1993) o TUANA (1989), (1993).

16 El problema se abordaba desde diversas disciplinas e incluso contribuyó al establecimiento de alguna de ellas como ciencia (PÉRZ SEDEÑO, 1998). Me limito aquí a la craneología de la que tambien los estudios son diversos, pero véase FEE (1980), GOULD (1997) y TUANA (1995).

17 Me ocupo aquí sólo de las mujeres, pero sucedía lo mismo con los índices utilizados para jerarquizar las razas. Véase GouLd (1997). 
el siguiente: de la "observación" de la inferioridad de las mujeres y las razas no europeas se extraía un conjunto de características que servía para clasificar a las mujeres y las razas no europeas como inferiores. Como dato auxiliar se utilizaba la semejanza entre los esqueletos de éstos y los primates.

Como buen empirista que comprueba sus hipótesis, Alexander Ecker (1868) constató que las mujeres y las razas no europeas no satisfacían la última de las características propuestas por Schaaffhausen, pero en vez de refutar la hipótesis y afirmar que las mujeres no estaban menos evolucionadas, concluyó lo contrario, argumentando que era un rasgo poseído por los niños. Finalmente, John Cleland (1882), rechazó los criterios utilizados por los anteriores craneólogos y utilizó uno nuevo, a saber, el ángulo que forma la línea de la base del cráneo con la columna vertebral: esta última medida apoyaba la creencia de que las mujeres, al igual que las razas no europeas, tenían un esqueleto infantil $y$, por consiguiente, estaban menos evolucionadas.

Aunque la exposición que acabo de hacer es muy somera ${ }^{18}$ resulta sumamente esclarecedora: todos coincidían en una hipótesis común con ligeras variaciones, a saber, considerar y aceptar que determinado(s) rasgo(s) del cráneo indica(n) menor evolución e inferioridad; además, a pesar de su afán por buscar evidencia empírica, cuando ésta contradice la hipótesis básica, no la abandonan, sino que sustituyen el criterio por otro que la avale. Pero además, este ejemplo, como otros muchos, muestra que no se trata tan sólo de un caso de mala ciencia que podría evitarse con la aplicación adecuada del buen método científico. En realidad, este ejemplo muestra la complejidad que hay en la relación evidencial de los supuestos básicos, que, incluyen en este caso la creencia en la inferioridad de las mujeres (o las razas no europeas).

Si seguimos con la vieja idea de que siempre que alguien tiene una hipótesis, $\mathrm{H}$, y encuentra evidencia en contra, $\mathrm{E}$, debe rechazar $\mathrm{H}$, nos encontraríamos ante "un caso más» de mala práctica científica. Sin embargo, la creencia en la inferioridad de las mujeres no era una hipótesis sometida a prueba, sino un supuesto básico, una verdad dada, un compromiso metafísico fundamentado en diversas teorías aceptadas por la comunidad científica ${ }^{19} \mathrm{y}$ reforzado por creencias (1978)

18 Para una exposición más amplia, remito a los textos mencionados en la nota 11 y FEE

19 Como, por ejemplo, la teoría de la evolución de Darwin. Véase A. GOMEZ (2000), Pérez Sedeño (1997), (1998a), Russet (1989), Schiebinger (1993) y TuANa (1993). 
y prácticas sociales y políticas ${ }^{20}$. Así pues, la ideología está dentro de los supuestos básicos, lo que dificulta enormemente distinguir tajantemente entre ciencia $\mathrm{y}$ valores $\mathrm{y}$, mucho menos, hablar de una ciencia libre de valores y neutra.

Las mujeres, filósofas o científicas, no deben limitarse al papel de vigilantes de la buena práctica científica, como propondría el feminismo liberal o el empirismo feminista ingenuo. Porque el problema no se localiza en las personas que practican la ciencia, en sus prejuicios, sino en ciertas características especiales de la producción del conocimiento. En concreto, en dos aspectos -nociones - fundamentales en esa producción del conocimiento científico: la objetividad y la autoridad. Dado su caracter fundamental, parece que sólo hay dos opciones: o las rechazamos completamente (lo que nos deja sin criterios de evaluación o aceptación) y consideramos que la elección entre hipótesis o teorías es una cuestión meramente política, o las aceptamos ciegamente ${ }^{21}$. Pero, una vez más, no parece conveniente plantear la cuestión en términos de alternativas excluyentes: hay aspectos de la objetividad científica que es deseable preservar, precisamente todos esos que conllevan creatividad individual en constante interacción práctica con la naturaleza, y crítica y validación por parte de la comunidad científica tanto de los métodos como de los resultados y conclusiones. ¿Qué deberíamos dejar de lado?

La crítica y reconsideración de la noción de objetividad ha sido una constante en la filosofía contemporánea pero está lejos de tener un significado claro, estable y simple ${ }^{22}$. En realidad, la noción de objetividad se construye en la ciencia en muchos niveles. En uno de ellos se asimila lo objetivo como el resultado o producto final frente al proceso por el que se ha llegado a él ${ }^{23} \mathrm{y}$ el

20 El auge de la craneología como teoría fundamentadora de la inferioridad social e intelectual de la mujer coincide con la primera ola del feminismo, el sufragismo, y con la lucha de las mujeres por acceder a las instituciones educativas superiores. Cf. PÉREZ SEDEÑo (2000), ROSSITER (1984).

21 Nótese que no sólo las feministas critican las nociones de objetividad y autoridad aparentemente inherentes a la ciencia y la tecnología: activistas medioambientalistas, antinucleares, partidarios de energías alternativas, pero tambien organizaciones religiosas fundamentalistas las han criticado.

22 Para un examen de distintas maneras de concebir la idea de objetividad, véase, por ejemplo Bustos (1999). Para el doble estándar que se usa con las epistemologías feministas con respecto a esta cuestión, véase E. LLOYD (1995).

23 Naturalmente paralelo a la separación entre contexto de justificación y de descubrimiento, sumamente denostada en nuestros días. 
uso social. De ese modo se diferencia entre el conocimiento producido, que conlleva decisiones y técnicas intelectuales y lógicas, pero libre de valores, y sus usos políticos y económicos, liberando de ese modo al científico de toda responsabilidad moral o social (aunque efectúen sus investigaciones gracias a los fondos militares).

En este sentido hay un aspecto de la noción de objetividad que plantea cuestiones distintas, a saber, la posición social, de autoridad, de los científicos. En una sociedad como la nuestra, los científicos aparecen en todos los terrenos, investidos de gran autoridad. En estos casos, cuando se aboga por la objetividad del científico, en realidad se está confundiendo con "pasividad política o ética». De hecho, cuando un científico renuncia a su posición privilegiada y a su "libertad de investigación" y se compromete políticamente, corre el grave riesgo de que se le acuse de haber dejado de ser «objetivo". Tenemos un ejemplo reciente en los recientes debates sobre ingeniería genética, clonación y alimentos transgénicos, en los que a los científicos que planteaban cuestiones éticas y políticas se les ha acusado de interferir en la objetividad de la ciencia, en su marcha inexorable.

Por otro lado, la "apoliticidad" de los científicos no es cierta, dado que se hallan en todos los niveles de la política pública, educativa, en los tribunales, etc. Y desde el momento en que se hallan en las instancias en que se toman las decisiones educativas, de financiación de proyectos de investigación, etc., no podemos decir que eso no tenga que ver con la práctica real de la ciencia. Por lo que respecta al científico como individuo que investiga y trabaja en un laboratorio, baste recordar que forma parte de una institución jerárquica, cuyo trabajo depende de decisiones políticas y económicas o de problemas que seguramente él no ha formulado sino que le vienen dados por parte de la institución. Debemos preguntarnos, pués, en qué medida puede el científico ser independiente de esos procesos políticos y económicos y cómo puede la ciencia ser autónoma con respecto a la organización social ${ }^{24}$.

En realidad, si nos damos cuenta de que el conocimiento científico está sumamente estructurado y organizado, veremos que cualquier sociedad intenta

24 Un caso reciente y próximo que permite comprobar cómo las decisiones político económicas afectan y son consustanciales a la propia ciencia es el de la evolución de la ciencia en España desde 1982; cf. SANZ (1997). 
producir el conocimiento que mejor satisfaga sus necesidades sociales, políticas y económicas, lo que determina qué tipo de cuestiones se pueden plantear y cuáles son los medios disponibles para ello. Esa estructuración y organización no se da sólo en el terreno institucional, sino tambien en el nivel de las creencias. Aun cuando cualquier científico admitirá como máxima suprema que todo es cuestionable, lo cierto es que hay creencias que hoy no son cuestionables aunque un día sí lo fueran y que en estos momentos podríamos considerar "evidentes", y otras sometidas a prueba ${ }^{25}$. Dado el carácter históricamente cambiante de nuestras creencias científicas, debemos abandonar la vieja idea de objetividad como propiedad absolutamente independiente de los seres humanos. Pero eso no implica el abandono de toda idea de objetividad (y acabar en el famoso todo vale) ni profesar un relativismo a ultranza. Podríamos considerar una noción de objetividad que se logra por el consenso obtenido por la comunidad científica que está formada, no lo olvidemos, por seres humanos, individuales que comparten una base experiencial o evidencial común. Pero para llegar a esa idea, debemos analizar aún otros aspectos de la noción de objetividad.

Otro de los niveles en que se construye la noción de objetividad tiene que ver con la separación - tan arraigada en la tradicción occidental- entre pensar (racional u objetivamente, se entiende) y sentir ${ }^{26}$. Cuando se exige objetividad se pretende separar tajantemente entre racionalidad científica y compromiso social o emocional. Se utiliza la idea de objetividad para devaluar posiciones expresadas con convicción emocional, justificando el dominio del "pensamiento" sobre el «sentimiento". Pero, ¿por qué una toma de decisión en que se tenga en cuenta el posterior o inmediato uso social, o los buenos sentimientos, es peor que una en la que supuestamente no se tengan en cuenta esos aspectos? ¿Por qué una decisión tomada de ese modo ha de ser necesariamente irracional o no objetiva? ¿No es posible elaborar criterios de evaluación que incluyan criterios éticos, políticos o sociales? Sobre todo, teniendo en cuenta muchas de las evaluaciones y decisiones tomadas en aras de la objetividad y la

25 Entre unas y otras hay toda la gama imaginable.

26 Creo que es conveniente recordar aquí cómo y dónde se origina esta distinción: en el Timeo de Platón. Cuando el demiurgo crea las almas y a cada una le asigna una estrella y luego se encarnan en hombres/varones (pues el universo platónico es en principio unisexual), aquellos que dominen sus pasiones y emociones y lleven una vida racional y justa volverán a sus estrellas; pero quienes se dejen dominar por las pasiones se reencarnarán en ... ¡mujeres! 
racionalidad científica (medicina nazi, desarrollo de la energía nuclear, etc.) parecería conveniente tener en cuenta ese tipo de criterios ${ }^{27}$.

Por último, como ya mencioné antes, la crítica feminista a la ciencia rechaza la diferenciación tajante, dicotómica y jerárquica, entre objetividad y subjetividad. Ese rechazo se basa en la sospecha, luego confirmada, de que el modelo de ciencia que manejan muchos científicos y la filosofía de la ciencia de la concepción heredada no es neutral: como todo modelo, surge de una tradición y está impregnada de sus valores. Es un modelo que se denomina masculino porque se asocia con características masculinas y las potencia, a la vez que suprime las asociadas con lo femenino ${ }^{28}$. Eso, junto con las barreras socioinstitucionales antes mencionadas explicaría la tradicional escasez de mujeres en ciencia y tecnología ${ }^{29}$. Así pués, hay que plantearse si realmente están excluidos los factores subjetivos del proceso de elaboración/construcción/obtención del conocimiento científico (lo que llevaría, en caso de contestación negativa, a replantearnos la relación entre el sujeto cognoscente y el objeto conocido). Pero también hay que examinar y cambiar, si es necesario, la naturaleza del sujeto de conocimiento: ¿quién es el sujeto cognoscente? ¿Es un sujeto individual, el sujeto cartesiano? ¿Es un sujeto situado y corporeizado? ¿Es un individuo en comunidad? ¿Es la comunidad?

Como mostraba perfectamente el ejemplo del debate craneológico, la identidad del sujeto de conocimiento no es algo ajeno a la ciencia y a la objetividad. El conocimiento científico está situado, el sujeto de conocimiento no es autónomo, desinteresado e imparcial (todos ellos sinónimos de "objetivo"). Pero el reconocimiento de cómo los valores forman parte de la práctica y progreso científicos no basta: hay que ver si son epistemológicamente relevantes.

27 La misma dicotomía que la anterior, pero a escala social, aparece reproducida en la distinción entre experto y no experto, pero su discusión aquí nos llevaría demasiado lejos.

28 Se suele poner como ejemplo del modo de hacer ciencia femenina a Barbara MCCLINTOCK, tal y como se describe su quehacer en Fox KELLER (1985). Curiosamente, cuando en 1983 se le concedió el premio Nobel de Medicina y Fisiología por el trabajo realizado varias décadas atrá se produjeron diversas reacciones interesantes. La wacademia» que en el pasado había considerado sus métodos y tesis como mínimo "heréticas" la acogió como "uno más". Como señaló Stephen JaY Gould (1984) su modo de trabajar implicándose en el objeto de estudio no era extraño: todos los buenos científicos procedían así.

29 Aunque el porcentaje de mujeres cursando carreras de ciencias es prácticamente igual al de varones en la mayoría de los casos (excepto en ingenierías), no sucede así en los puestos de trabajo y es claramente inferior en los puestos directivos. Véase PérEZ SEDEÑo 1995a, $1995 \mathrm{~b}$. 
Para ello es conveniente distinguir dos aspectos: por un lado, el carácter situado del sujeto, es decir, su localización histórica, social, cultural (lo que incluiría, obviamente, sexo, raza, clase...). Y, por otro, la relación entre el sujeto cognoscente y el objeto de conocimiento, en la cual hay una serie de aspectos usualmente considerados subjetivos: compromisos, deseos, intereses, pero también el hecho de que ese sujeto tiene un cuerpo con/de cierta naturaleza. Esta distinción es analíticamente conveniente, aunque es difícil distinguir ambos tipos de subjetividad, dado que los compromisos, deseos, e incluso cómo experimentamos nuestro propio cuerpo están social, histórica y culturalmente constituídos.

Al hablar de obetividad en el sentido tradicional, se presenta la ciencia como una actividad hostil o impropia de las mujeres, pues los métodos de la ciencia chocan con la construcción social de la feminidad. Si convirtiéramos, en cambio, el sujeto de conocimiento científico, en un ser participativo, que se implica emocionalmente y se compromete en lo que hace (características, al parecer, femeninas), cambiaría nuestra percepción del conocimiento. Como Lorraine Code ha señalado, no todo conocimiento es conocimiento proposicional, el único que parece tenerse en cuenta cuando se habla de separación entre objetividad/subjetividad. El conocimiento exige aprendizaje constante, interpretación a varios niveles, de diversos grados, es, en suma, algo mucho más complejo que lo que se recoje en el esquema «S sabe que $p »{ }^{30}$. Sin embargo, la epistemología y el modo de enseñar ciencia en nuestras universidades, olvida con frecuencia que no todo conocimiento es proposicional, que hay cosas que se aprenden haciendo y experimentando, que hay otras formas de conocimiento que, por cierto, no es privativo de las mujeres.

El sujeto que aparece en el modelo proposicional de conocimiento es un sujeto: libre de todo lo aprendido, de las necesidades de su cuerpo, de todo influjo emocional, estético, social, etc. Este sujeto es el sujeto cartesiano. La

30 El modelo que utiliza Code se asemeja al que utilizan muchos profesionales de la medicina que se dedican a la atención primaria: médicos, ATS, etc. Ellos son entrenados en teorías y técnicas específicas y observan a través de instrumentos más o menos sofisticados, pero también tocan y aprenden del tacto, escuchan, no sólo a través de instrumentos, sino, además al paciente, etc. pues aprendan a través de ellos. Como todo buen profesional sabe, dice Code, un tratamiento tiene tanto más éxito cuanto más sabe el paciente de su enfermedad y su terapia; cuando se establece un clima de comunicación y confianza entre enfermo y profesionales, en el que están implicados el intelecto, pero, también, la imaginación y la emoción. 
interpretación que las feministas han hecho de la filosofía cartesiana es diferente. Autoras como Ruth Perry (1986) mantienen el caracter inicialmente positivo y feminista del dualismo cartesiano: si el cuerpo es una máquina, no hay base para sustentar la idea aristotélica de la inferioridad intelectual de la mujer y si la mente actúa independientemente del cuerpo, no se puede utilizar éste, como base de la inferioridad. Otras feministas, como Genevieve Lloyd, afirman, en cambio, que los efectos, fueron a la larga, negativos, pues en la distinción mente/cuerpo se halla la semilla de la división sexual del trabajo. De hecho, creo que al separar la mente del cuerpo se creó el ideal de una razón pura, sumamente abstracta que no tiene una localización espacio-temporal y completamente separada del cuerpo, esto es, de la historia y de la sociedad. En cualquier caso, ese ideal se convirtió en uno de los pilares fundamentales de la ciencia y de la epistemología modernas, sólo cuestionado muy recientemente. Una filosofía que separa tajantemente los requisitos de búsqueda de la verdad de la vida cotidiana fundamenta las dicotomías jerárquicas producción de conocimiento/uso de éste, experto/no-experto, objetivo/subjetivo y refuerza la distinción existente entre masculino/femenino, vida masculina/vida femenina.

Percepción y cognición son idénticas en todos los sujetos y lo demás, creencias personales, deseos, configuración corporal, etc. es nocivo, contaminante o, en el mejor de los casos, irrelevante. Naturalmente, eso contradice uno de los postulados feministas, a saber, la relevancia del género/sexo en todos los niveles, dado que las diferentes posiciones sociales que ocupan hombres y mujeres estructuran todo tipo de relaciones humanas. Esas relaciones de interdependencia se dan también y son cruciales en las deliberaciones morales, políticas y científicas. De hecho, el examen cuidadoso de la práctica científica del tipo efectuado no sólo por las feministas sino por historiadores, sociólogos y filósofos de la ciencia contradice esa visión idealista. Como dice Polanyi: «se considera que la ciencia está objetivamente establecida a pesar de sus orígenes pasionales ... Debe estar ya claro que disiento de esa creencia y... quiero ocuparme explícitamente de las pasiones en la ciencia. Quiero mostrar que las pasiones científicas no son un mero subproducto psicológico, sino que desempeñan una función lógica que constituye un elemento indispensble en la ciencia” ${ }^{31}$. Esos

31 Polany (1958), pág. 134. La lista de autores sería enorme, por lo que me he limitado a este autor. 
trabajos indican que es mejor optar por un modelo dinámico que preste atención a los individuos como participantes/componentes de una comunidad. Lo que conduce ineludiblemente al rechazo de la dicotomía ciencia/política, dado que las situaciones/localizaciones del científico o la científica pueden ser epistémicamente significativas para cómo practica la ciencia.

La comunidad o comunidades a la que pertenece el científico o la científica son relevantes epistémicamente, pues sus valores, sus supuestos básicos, estarán presentes en sus investigaciones. Vimos cómo sucedía en el caso de la craneología, pero hay otros muchos. Por ejemplo, diversas autoras ${ }^{32}$ han mostrado cómo la entrada en la primatología de mujeres con preocupaciones feministas varió la idea de universalidad del liderato masculino en las jerarquías de dominación entre los primates. Otras ${ }^{33}$ modificaron el modelo que atribuía importancia evolutiva a la caza (masculina) frente a la recolección (femenina). La conciencia política - feminista - de estas mujeres les hace replantearse las teorías en las que han sido entrenadas y plantear alternativas. Pero esas mujeres también pertenecen a determinadas comunidades científicas - la de la primatología, en un caso, la evolutiva, en otro- de las que comparten supuestos básicos, valores, etc. Y las alternativas propuestas no son correcciones feministas, sino que, debido a sus creencias feministas, pueden considerar la evidencia de otro modo. Lo interesante es que su pertenecia a la comunidad científica y a la no científica - los valores y creencias adquiridos en ellas- permiten crear una alternativa mejor porque discrimina y atiende a la complejidad de las especies de primates en un caso, porque incorpora a ambos sexos en el proceso evolutivo, en el otro.

Esos ejemplos muestran la miopía de las concepciones tradicionales del sujeto cognoscente cartesiano y de la objetividad. Según el modelo $\mathrm{S}$ sabe que p", no es necesario saber nada acerca de $S$, pero los ejemplos que he puesto (y otros muchos) muestran al menos dos cosas: lo importante que es saber algo acerca de $S$, aunque en muchas ocasiones resulte muy difícil determinar qué relaciones y comunidades son epistémicamente significativas; y cuán absurdo es pretender tener una ciencia neutral y libre de valores. El problema, y el reto, es crear un modelo que, reconociendo el papel de los valores contextuales (describiendo), permita evaluar y decidir entre teorías y prácticas científicamente aceptables (es decir, puede establecer criterios normativos).

32 HaRaWAy (1989), LeIBOWITZ (1975), ROSSER (1992).

33 SLOCUM (1974), ZiHLMAN (1981), (1985). 
Las soluciones propuestas son diversas y ya hemos apuntado algunas de ellas. El empirismo contextual propuesto por Helen Longino considera que los argumentos que aducimos a favor de ciertas evidencias dependen del contexto y que consideramos que ciertos datos constituyen evidencia a favor o en contra de ciertas hipótesis sólo con respecto a otras hipótesis o supuestos previos. Es decir, la construcción del conocimiento es una práctica social; por eso, la investigación conlleva valores e ideología, pero eso no supone que haya que tolerar de forma indiscriminada las preferencias subjetivas individuales. Lo que confiere objetividad a los productos de la investigación científica es la crítica social junto con la evidencia empírica. Es decir, se entiende que el conocimiento científico es, sobre todo, una práctica que tiene lugar en un contexto particular y es evaluado con respecto a fines particulares: es decir, se considera la ciencia como proceso y actividad de comunidades científicas insertas en contextos sociohistóricos concretos en cuyo seno encontramos valores personales, sociales y culturales, preferencias de grupos o individuales, de tipo cultural, social, que inciden en diversos modos y grados o que pueden incidir sobre la práctica científica.

La objetividad se entiende como una función de la práctica de la comunidad de la cual forma parte, precisamente, la crítica intersubjetiva y que evalúa los supuestos implícitos en las observaciones, en los razonamientos, en la consideración de plausibilidad de cierta hipótesis y no otra o en la consideración de que ciertos métodos, y no otros, son los adecuados para resolver ciertos problemas (teniendo en cuenta que la influencia de valores externos o contextuales a veces distorsiona la actividad científica y a veces no). Si queremos descubrir esos supuestos deberemos contar con tantos puntos de vista como sea posible y la ciencia occidental no puede lograr esa interacción completa, no puede lograr esa «objetividad", si deja de lado a la mitad de la humanidad.

La comunidad científica maneja criterios internos normativos respecto a lo que es científicamente aceptable y evaluativos respecto a fines y objetivos en los que otros componentes de la ciencia (razonamiento, observación, experimentación) tienen también importancia. Por lo que se refiere a los criterios evaluativos, $\mathrm{H}$. Longino ${ }^{34}$ ha propuesto una serie de virtudes epistemológicas, valores o criterios, alternativos al conjunto propuesto por Kuhn (1977) y que tienen la característica de poder ser internos o externos. Dichos criterios o virtudes son 
la adecuación empírica, la novedad, la heterogeneidad ontológica, la interacción mutua, la aplicabilidad a las necesidades humanas y la difusión (o expansión) de poder. Se considera que la adecuación empírica es un valor cognitivo por su capacidad para guiar la investigación. Pero desde el feminismo se considera además, que debe servir también para revelar la aparición del género en los fenómenos y los segos de género ${ }^{35}$, en la explicación que se da de dichos fenómenos, por lo que adquiriría además la consideración de contextual. La virtud (o el criterio) de la novedad ${ }^{36}$ recomienda las teorías y modelos que se apartan de las teorías aceptadas. La mutualidad de interacción descarta los modelos de un solo factor causal en favor de modelos dinámicamente interactivos - todos los factores influyen en los demás y no hay uno sólo dominante- lo que permite dar cuenta de fenómenos tecnocientíficos más complejos, como recientes trabajos en bioquímica celular y neurociencia muestran. Las ventajas de la difusión de poder, de sobra conocidas y postuladas desde diversos ámbitos, no sólo el feminista, permite dar preferencia a programas de investigación que incorporan relaciones mutuas, no de dominador/subordinado y, por tanto, propicia el control democrático de la investigación.

Este tipo de filosofía de la ciencia, la epistemología feminista, considera, pues, que las consideraciones ideológico-políticas son constricciones relevantes en el razonamiento y la interpretación que conforman el contenido del conocimiento. Esos valores no son pura y únicamente cognitivos $y$, tal y como se usan en ciertos contextos de argumentación científica, de evaluación, lo que hacen es importar valores sociopolíticos a esos contextos.

He pretendido mostrar que el camino recorrido por la filosofía de la ciencia desde la publicación de la obra de Kuhn ha experimentado cambios de contenido y de perspectivas, a muchos de los cuales el pensamiento feminista ha contribuído de forma importante. Se ha pasado de una consideración y análisis exclusivamente de los resultados a una visión que capte los procesos y actividades abarcando la mayor cantidad posible de tipos de problemas y perspectivas, de una separación tajante entre dos contextos a una pluralidad y trasva-

35 Que tambien pueden ser de otro tipo: racistas, clasistas...

36 Este criterio "contradice» aparentemente el kuhniano de coerencia externa, es decir, el acuerdo de la nueva hipótesis o teoría propuesta con otras ya aceptadas. Sin embargo, muchos casos históricos muestran que es fundamental para abandonar prejuicios y para el progreso de la ciencia. Para un caso de no aplicación del criterio de novedad y sus consecuencias, véase, por ejemplo, Pérez Sedeño (en prensa). 
se entre ellos ${ }^{37}$. Hoy en día, por ejemplo, no es posible enfrentar el análisis de la estructura de teorías sin tener en cuenta los resultados de los estudios históricos o sociológicos. De igual modo, resulta imposible desarrollar estudios sociológicos al margen de las elucidaciones conceptuales sobre las teorías o prácticas empleadas.

Esa consideración de la ciencia en cuanto actividad permite tener en cuenta aspectos que de otro modo se obviarían: la práctica científica efectuada por individuos, con un determinado sexo, que colaboran entre sí, de una manera socialmente organizada y que se desarrolla en contextos sociales, políticos e históricos con los que interactúa; pero también que la actividad científica tiene ciertos fines, uno de los cuales es, sin duda, la obtención de conocimiento. Esta multiplicidad de aspectos debe quedar reflejada en una materia que pretende ser una reflexión crítica sobre la ciencia y que debe incorporar la tecnología, dada la cada vez mayor interdependencia de ambas. Sólo así ganaremos todos.

\section{Referencias bibliográficas}

Alcala, P. y Pérez Sedeño, E. (eds.) (2000): Ciencia y género, Madrid, Ed. Complutense.

Ambrogi, A. (ed.) (1999): Filosofia de la ciencia: el giro naturalista, Universitat de les Illes Balears.

BLEIER, R. (1979): “Social and political bias in science:an examination of animal studies and their generalization to human behaviors and evolution", en R. Hubbard y M. Lowe (eds.), Genes and Gender II, Nueva York, Gordian Press.

- (1984): Science and Gender, Nueva York, Pergamon Press.

Bustos Guadaño, E. (1999): «Objetividad", en L. Villoro (ed.) El conocimiento, en Enciclopedia iberoamericana de la filosofia, Madrid, Trotta.

Caplan, A. L. (ed.) (1978): The Sociobiological Debate, Nueva York, Harper \& Row. Cleland, J. (1882): The relation of Brain to Mind, Galsgow, James Maclehousse \& Sons.

CODE, L. (1991): What can she know? Feminist Theory and the Construction of Knowledge, Londres/Ithaca, Cornell Univ. Press.

37. J. ECHEVERRIA (1995). 
De MORA Charles, M. S. (ed.) (1998): La construcción de la ciencia. Abstracción y visualización, San Sebastián, New Books Ediciones.

DURAN, Jane (1998): Philosophies of Science/Feminist Theories, Westview Press.

ECHEVERRIA, J. (1995): Filosofia de la ciencia, Madrid, Akal.

ECKER, A. (1868): “On a Characteristic peculiarity in the Form of the Female Skull and Its Significance for Comparative Anthropology",Anthropological Review, 6, 350-356.

FAUSTO-STERLING, A. (1985): Myths of Gender, Basic Books.

FEE, E. (1978): Science and the "Woman Question" 1860-1920. A Study of English Scientific Periodicals, Tesis doctoral presentada en la Universidad de Princeton

- (1980): «Nineteenth Century Craniology: The Study of the Female Skull»; Bulletin of the History of Medicine,53, 415-433.

GIERE, R. (1985): “Philosophy of Science Naturalized», Philosophy of Science, 52, págs. 331-356. Trad. Esp. En Ambrogi (1999).

GOMez RodrfGuez, A. (2000): "La hembra que nunca evolucionó» en ALCALA y PÉREZ SEDEÑo (eds.)

- (Ed.),(1998) La construcción social de lo femenino, Ed. De la Universidad de La Laguna, Tenerife.

González Garcia, M. y Pérez Sedeño, E. (2000): "Ciencia, tecnología y género", Revista Iberoamericana de Educación.

Gould, S. J. (1981): La falsa medida del hombre, BarcelonaCrítica. Hay una nueva edición aumentada en la colección Drakontos de la ed. Crítica, Barcelona, 1997.

- (1984): «Barbara McClintock, Nobel Prize», New York Review, 26 de marzo.

Haraway, D. (1989): Primate Visions, Londres, Routledge.

- (1991): Simians, Cyborgs, Women, Londres, Routledge.

- (1995) «Situated Knowledge: The Science Question in Feminism and the Privilege of Partial Perspective», en Feenberg y Hannay (eds.).

- (1997): Modest_Witness@Second_Millennium.FemaleMan@_Meets_OncoMouse ${ }^{\mathrm{TM}}$, Nueva York, Routledge.

HubBard, R. (1990) The Politics of Women's Biology, New Brunswick, Rutgers Univ. Press. JoRdanova, L. (1990); Sexual visions, University of Wisconsin Press.

KEller, E. Fox (1983): A Feeling for the Organism: The Life and Work of Barbara McClintock, Nueva York, W. H. Freeman. (Hay trad. cast., Seducida por lo vivo, Ed. Fontalba, 1985). 
- (1985): Reflexions on Gender and Science, New Haven, Yale Univ. Press. Hay trad. esp.Refleciones sobre ciencia y género, Valencia, Edicions Alfons El Magnànim, 1989

- (1995) Refiguring Life, Nueva York, Columbia Univ. Press. University of Chicago Press (Hay traducción al español, La tensión esencial, F.C.E., 1982.

KLEE, R. (1997):Introduction to philosophy of Science. Cutting Nature at its seams, Nueva York/Oxford, oxford University Press.

KuHN, T. S. (1962): The Structure of Scientific Revolutions, Chicago, 1962. Trad. esp. La estructura de las revoluciones cientificas, México, F.C.E., 1968.

- (1977): "Objectivity, Values and Theory Choice», en The Essential Tension, Chicago, The Chicago Univ. Press. Trad. Cast. La tensión esencial México, F.C.E., 1982.

LaQueur, T. (1990); Making Sex, Harvard University Press. Trad. esp. La construcción del sexo. Cuerpo y género desde los griegos hasta Freud, Valencia, Cátedra.

Leibowitz, L. (1975):Females, Males, Families: A Biosocial Approach, Belmosnt, Duxbury.

Lewontin, R. C., Rose, S. \& KAMIN, L. J. (1984): Not in our genes: Biology, ideology and human nature, Nueva York, Pantheon. Trad. esp. No está en los genes. Racismo, genética e ideología, Barcelona, Crítica, 1987.

LloYd, E. (1995): "Objectivity and the Double Standard for Feminist Epistemologies", Synthese, 104:3, págs. 352-381.

LLOYD, G. (1984): The Man of Reason: "Male» and "Female» in Western Philosophy, Minneapolis.

LongINo, H., Science as Social Knowledge, Princeton, Princeton Univ. Press, 1990.

- (1996): «Cognitive and non-cognitive values in science: rethinking the dichotomy", en L. H. Nelson y J. Nelson (eds.).

- (1999): "Conocimiento social”, en A. Ambrogi (ed.).

- (2000): “Feminist Epistemologies» en J. Greco y E. Sosa (eds.), Blackwell Guide to Epistemology, Oxford, Blackwell.

LoNGINO, H. y Doell, R.(1983): «Body, bias and behaviour», Signs, 9(2): 206-227.

LOPEZ DE LA VIEJA, T. (ed.) (2000): Feminismo: del pasado al presente, Ediciones de la Universidad de Salamanca.

MARTIN, E. (1991): "Egg and Sperm", Signs,:: Journal of Women in Culture and Society, 16,3 .

Nelson, L. H. (1990): Who Knows?From Quine to a Feminist Empiricism, Filadelfia, Temple University Press. 
Nelson, L. H. y Nelson, J. (eds.) (1996): Feminis, Science and Philosophy of Science, Kluwer Academic Pub.

POlanyi, M. (1958): Personal Knowledg. Towards a Post-critical Philosophy, Londres, Routledge \& Kegan Paul.

Pérez SedeÑo, E.(1995): «Filosofia de la ciencia y feminismo: Interseccuón y convergencia", Isegoria, vol. 12.

- (1995a): «Scientific academic careers of women in Spain: History and Facts», en Proceedings of the VIth ILS Conference, Franckfort, KY.

- (1995b): "La sindrome de l'Snark i altres històries", en Debates sobre Mujer y Ciencia, Cuadernos del Observatorio de La comunicacion cientifica, Barcelona, 1995.

- (1997): «La retórica de la ciencia y el estudio de las diferencias sexuales", Revista de Filosofia, México U.N.A.M.

- (1998): «Los valores de la invención», en De Mora (ed.).

- (1998a): "Las amistades peligrosas» en A. Gómez (ed.).

- (1998b) «Factores contextuales, tecnología y valores: ¿desde la periferia», en P. Martínez Freire (ed.) Filosofia actual de la ciencia, suplm. No 3 de Contrastes. Revista Interdisciplinar de Filosofia.

- (2000): «¿El poder de una ilusión? Ciencia, género y feminismo», en López de la Vieja (ed.)

- (En prensa): «Cambio de valores en tiempos de guerra», en J. Echeverría y A. Ibarra (eds.), Ciencia, guerra y valores, Akal.

PerRY, R. (1986): The Celebrated Mary Astell: An Early English Feminist, Chicago, Chicago Univ. Press.

QUINE, W. V. O. (1969): «Epistemology Naturalized», en Ontological Relativism and Other Essays, Nueva York, Columbia University Press. Hay trad. Esp. La relatividad ontológica y otros ensayos, Madrid, Tecnos, 1974.

RoONEY, P. (1991): «Methodological Issues in the Construction of Gender as a Meaningful Variable in Scientific Studies of Cognition", en D. Hull, M. Forbes y R. Burian (eds.), PSA 1994, vol. 2 (Philosophy of Science Association, 1995).

ROSSER, Sue V. (1992): "Are there feminist methodologies appropriate for the natural sciences and do they make a difference?», Women's Studies International Forum, Vol. 15, Nos. 5/6, 5335-560.

Rossiter, M., (1984): Women Scientists in America. Struggles and Strategies to 1940, The John Hopkins University Press. 
RuSE, M. (1980): Sociobiology: Sense or Nonsense? Dordrecht, Holland, Reidel pub. Co. Trad. esp. Sociobiologia, Madrid, Cátedra, 1983.

RussetT, C.E. (1989): Sexual Science. The Victorian Construction of Woomanhood, Cambridge, Harvard Univ. Press.

SANZ MENÉNDEZ, L. (1997): Estado, ciencia y tecnologia en España: 1939-1997, Madrid, Alianza.

SAYErs, J (1982): Biological Politics: Feminist and Anti-Feminist Perspectives, Nueva York, Tavistock.

SCHAaffHausen, H. (1868): "On the primitive Form of the Human Skull", Anthropological Review, 6, 412-431.

SCHIEBINGer, L.(1989), The Mind Has No Sex? Women in the Origins of Modern Science. Cambridge, Mass/Londres, Harvard University Press.

- (1993): Nature's Body: Gender in the Making of Modern Science, Boston, Beacon.

SLOCUM, S.L. (1974): "Woman the Gatherer: Male Bias in Anthropology", en S. Jacobs (ed.), Women in Perspective: A Guide for Cross-Cultural Studies, Urbana, Univ. Of Illinois Press.

SмrTh, D. (1988): The Everyday World as Problematic: A Feminist Sociology, Boston, MA, Northeastern Univ. Press.

SOLf́s SANTOS, C. (1998): Alta tension: filosofia, sociología e historia de la ciencia, Barcelona, Paidós.

Tuana, N. (1989): Feminism and Science, Bloomington, Indiana Univ. Press.

- (1992): Woman and the History of Philosophy. Nueva York, Paragon House.

- (1993): The Less Noble Sex, Bloomington, Indiana University Press.

- (1995): «The values of science: empiricism from a Feminist perspective», Synthese, 104:3, págs. 441-477.

ZIHLMAN, A. (1981): «Women as Shapers of Human Adaptation», en F. Dahlberg (ed.) Woman the Gatherer, New Haven, Yale University Press.

- (1985): «Gathering Stories for Hunting Human Nature», Feminist Studies, 11, págs. 365-377. 